\title{
On Kirchhoff Problems Involving Critical Exponent and Critical Growth
}

\section{Mohammed El Mokhtar O. El Mokhtar, Zeid I. Almuhiameed}

Department of Mathematics, College of Science, Qassim University, Buraidah, Saudi Arabia

Email:med.mokhtar66@yahoo.fr, M.labdi@qu.edu.sa,ksapr006@yahoo.com,zimhiemied@qu.edu.sa

How to cite this paper: El Mokhtar O. El Mokhtar, M. and Almuhiameed, Z.I. (2019) On Kirchhoff Problems Involving Critical Exponent and Critical Growth. Journal of Applied Mathematics and Physics, 7, 781-792.

https://doi.org/10.4236/jamp.2019.74053

Received: March 9, 2019

Accepted: April 9, 2019

Published: April 12, 2019

Copyright $\odot 2019$ by author(s) and Scientific Research Publishing Inc. This work is licensed under the Creative Commons Attribution International License (CC BY 4.0).

http://creativecommons.org/licenses/by/4.0/

\begin{abstract}
In this paper, we establish the existence of multiple solutions to a class of Kirchhoff type equations involving critical exponent, concave term and critical growth. Our main tools are the Nehari manifold and mountain pass theorem.
\end{abstract}

\section{Keywords}

Kirchhoff Equations, Critical Growth, Nehari Manifold, Mountain Pass Theorem

\section{Introduction}

In this paper, we consider the multiplicity results of nontrivial solutions of the following Kirchhoff problem

$$
\begin{cases}L_{a, p} u=|x|^{-b p^{*}}|u|^{p^{*}-2} u+\lambda k|u|^{q-2} u & \text { in } \Omega, \\ u=0 & \text { on } \partial \Omega\end{cases}
$$

where $\quad L_{a, p} v:=-M\left(\int_{\Omega}|x|^{-a p}|\nabla v|^{p} \mathrm{~d} x\right) \operatorname{div}\left(|x|^{-a p}|\nabla v|^{p-2} \nabla v\right), \quad \Omega \quad$ is a smooth bounded domain of $\mathbb{R}^{N}, 1<p<N, 0<a \leq \frac{N-p}{p}, \quad p^{*}=\frac{N p}{N-d p}$ with $d=1+a-b, a \leq b<a+1,1<q<2, \lambda$ is a real parameter, $k \in\left(\mathcal{D}_{a}^{1, p}\right)^{-1} \cap C(\bar{\Omega})$ with $\left(\mathcal{D}_{a}^{1, p}\right)^{-1}$ is the topological dual of $\mathcal{D}_{a}^{1, p}(\Omega)$ satisfying suitable conditions and $M: \mathbb{R}^{+} \cup\{0\} \rightarrow \mathbb{R}^{+}$continuous function.

The original one-dimensional Kirchhoff equation was introduced by Kirchhoff [1] in 1883 as an generalization of the well-known d'Alembert's wave equation: 


$$
\varrho \frac{\partial^{2} u}{\partial t^{2}}-\left(\frac{P_{0}}{h}+\frac{E}{2 L} \int_{0}^{L}\left|\frac{\partial u}{\partial x}\right|^{2} \mathrm{~d} x\right) \frac{\partial^{2} u}{\partial x^{2}}=g(x ; u) .
$$

His model takes into account the changes in length of the strings produced by transverse vibrations. Here, $L$ is the length of the string; $h$ is the area of the cross section; $E$ is the Young modulus of the material; $\varrho$ is the mass density and $P_{0}$ is the initial tension.

In recent years, the existence and multiplicity of solutions to the nonlocal problem

$$
\begin{cases}-\left(a+b \int_{\Omega}|\nabla u|^{2} \mathrm{~d} x\right) \Delta u=g(x ; u) & \text { in } \Omega, \\ u=0 & \text { on } \partial \Omega\end{cases}
$$

has been studied by various researchers and many interesting and important results can be found. In [1], it was pointed out that the problem (1.2) models several physical systems, where $u$ describes a process which depends on the average of it self. Nonlocal effect also finds its applications in biological systems. The movement, modeled by the integral term, is assumed to be dependent on the energy of the entire system with $u$ being its population density. Alternatively, the movement of a particular species may be subject to the total population density within the domain (for instance, the spreading of bacteria) which gives rise to equations of the type

$$
u_{t}-m\left(\int_{\Omega} u \mathrm{~d} x\right) \Delta u=h
$$

For the multiplicity, certain chemical reactions in tubular reactors can be mathematically described by a nonlinear two-point boundary-value problem and one is interested if multiple steady-states exist, for a recent treatment of chemical reactor theory and multiple solutions and the references therein. Bonanno in [2] established the existence of two intervals of positive real parameters for which the functional has three critical points whose norms are uniformly bounded in respect to belonging to one of the two intervals and he obtained multiplicity results for a two point boundary-value problem.

For instance, positive solutions could be obtained in [3] [4] [5]. Especially, Chen et al. [6] discussed a Kirchhoff type problem when

$$
g(x ; u)=f(x) u^{p-2} u+\lambda g(x)|u|^{q-2} u
$$

where $1<q<2<p<2^{*}=2 N /(N-2)$ if $N \geq 3,2^{*}=\infty$ if $N=1,2, f(x)$ and $g(x)$ with some proper conditions are sign-changing weight functions. And they have obtained the existence of two positive solutions if $p>4,0<\lambda<\lambda_{0}(a)$.

Researchers, such as Mao and Zhang [7], Mao and Luan [8], found sign-changing solutions. As for in nitely many solutions, we refer readers to [8] [9]. He and Zou [9] considered the class of Kirchhoff type problem when $g(x ; u)=\lambda f(x ; u)$ with some conditions and proved a sequence of i.e. positive weak solutions tending to zero in $L^{\infty}(\Omega)$. 
In the case of a bounded domain of $\mathbb{R}^{N}$ with $N \geq 3$, Tarantello [10] proved, under a suitable condition on $f$, the existence of at least two solutions to (1.2) for $a=0, b=1$ and $g(x ; u)=|u|^{\frac{4}{N-2}} u+f$.

Before formulating our results, we give some definitions and notation.

The space $\mathcal{D}_{a}^{1, p}(\Omega)$ is equiped with the norm

$$
\|u\|=\left(\int_{\Omega}|x|^{-a p}|\nabla v|^{p} \mathrm{~d} x\right)^{1 / p}
$$

wich equivalent to the norm

$$
\|u\|_{r, \alpha}=\left(\int_{\Omega}|x|^{-\alpha}|u|^{r} \mathrm{~d} x\right)^{1 / r},
$$

Let $S_{a, p}$ be the best constant of the weighted Caffarelli-Kohn-Nirenberg type,

$$
S_{a, p} \leq \frac{\int_{\Omega}|x|^{-a p}|\nabla v|^{p} \mathrm{~d} x}{\left(\int_{\Omega}|x|^{-b p^{*}}|u|^{p^{*}} \mathrm{~d} x\right)^{p / p^{*}}}
$$

Since our approach is variational, we define the functional $I$ on $\mathcal{D}_{a}^{1, p}(\Omega)$ by

$$
I(u)=(1 / p) \hat{M}\left(\|u\|^{p}\right)-\left(1 / p^{*}\right) \int_{\Omega}|x|^{-b p^{*}}|u|^{p^{*}} \mathrm{~d} x-(\lambda / q) \int_{\Omega} k|u|^{q} \mathrm{~d} x,
$$

A point $u \in \mathcal{D}_{a}^{1, p}(\Omega)$ is a weak solution of the Equation (1.1) if it is the critical point of the functional $I$. Generally speaking, a function $u$ is called a solution of (1.1) if $u \in \mathcal{D}_{a}^{1, p}(\Omega)$ and for all $v \in \mathcal{D}_{a}^{1, p}(\Omega)$ it holds

$$
M\left(\|u\|^{p}\right) \int_{\Omega}|x|^{-a p}|\nabla u|^{p-2} \nabla u \nabla v \mathrm{~d} x-\int_{\Omega}|x|^{-b p^{*}}|u|^{p^{*}-2} u v \mathrm{~d} x-\lambda \int_{\Omega} k|u|^{q-1} u v \mathrm{~d} x=0 .
$$

with $M$ is incresing and verifying

$$
0<M_{0}(t)= \begin{cases}M(t) & \text { if } 0 \leq t \leq t_{0} \\ M_{0}(t) & \text { if } t \geq t_{0}\end{cases}
$$

Throughout this work, we consider the following assumption:

(K) There exist $v_{0}>0$ and $\delta_{0}>0$ such that $k(x) \geq v_{0}$, for all $x$ in $B\left(0,2 \delta_{0}\right)$.

Here, $B(a, r)$ denotes the ball centered at $a$ with radius $r$.

In our work, we research the critical points as the minimizers of the energy functional associated to the problem (1.1) on the constraint defined by the Nehari manifold, which are solutions of our system.

Let $\Lambda_{0}$ be positive number such thatwhere

$$
\Lambda_{0}=\left(\frac{p^{*}-p}{p^{*}-q}\right)\left(\frac{p-q}{p^{*}-q}\right)^{\left(\frac{p-q}{p^{*}-q}\right)} M_{0}^{\frac{1}{p-q}}|k|_{\infty}^{-1}\left(S_{a, p}\right)^{\frac{p^{*}(p-q)}{p\left(p^{*}-p\right)}} .
$$

Now we can state our main results.

Theorem 1. Assume that $1<q<2$, and $(K)$ satisfied and $\lambda$ verifying $0<\lambda<\Lambda_{0}$, then the problem (1.1) has at least one positive solution. 
Theorem 2. In addition to the assumptions of the Theorem 1 , if $(K)$ hold and $M_{0}>\frac{p}{p^{*}}$ then there exists $\Lambda_{1}>0$ such that for all $\lambda$ verifying $0<\lambda<\min \left(\Lambda_{0}, \Lambda_{1}\right)$ the problem (1.1) has at least two positive solutions.

Theorem 3. In addition to the assumptions of the Theorem 2, there exists $\Lambda_{2}>0$ such that for all $\lambda$ verifying $0<\lambda<\min \left(\Lambda_{1}, \Lambda_{2}\right)$, then the problem (1.1) has at least two positive solution and two opposite solutions.

This paper is organized as follows. In Section 2, we give some preliminaries. Section 3 and 4 are devoted to the proofs of Theorems 1 and 2. In the last section, we prove the Theorem 3 .

\section{Preliminaries}

Definition 1. Let $c \in \mathbb{R}, \mathrm{E}$ a Banach space and $I \in C^{1}(E, \mathbb{R})$.

1) $\left(u_{n}\right)_{n}$ is a Palais-Smale sequence at level $c$ (in short $(P S)_{c}$ ) in $E$ for $I$ if

$$
I\left(u_{n}\right)=c+o_{n}(1) \text { and } I^{\prime}\left(u_{n}\right)=o_{n}(1),
$$

where $o_{n}(1)$ tends to 0 as $n$ goes at infinity.

2) We say that $I$ satisfies the $(P S)_{c}$ condition if any $(P S)_{c}$ sequence in $E$ for $I$ has a convergent subsequence.

Lemma 1. [11] Let $X$ Banach space, and $I \in C^{1}(X, \mathbb{R})$ verifying the Palais-Smale condition. Suppose that $I(0)=0$ and that:

1) there exist $R>0, r>0$ such that if $\|u\|=R$, then $J(u) \geq r$;

2) there exist $\left(u_{0}\right) \in X$ such that $\left\|u_{0}\right\|>R$ and $I\left(u_{0}\right) \leq 0$;

Let

$$
C=\inf _{\gamma \in \Gamma} \max _{t \in[0,1]}(I(\gamma(t)))
$$

where

$$
\Gamma=\left\{\gamma \in C([0,1] ; X) \text { such that } \gamma(0)=0 \text { et } \gamma(1)=u_{0}\right\} \text {, }
$$

then $c$ is critical value of $I$ such that $c \geq r$.

\section{Nehari Manifold [12]}

It is well known that the functional $I$ is of class $C^{1}$ in $\mathcal{D}_{a}^{1, p}(\Omega)$ and the solutions of (1.1) are the critical points of $I$ which is not bounded below on $\mathcal{D}_{a}^{1, p}(\Omega)$. Consider the lowing Nehari manifold

$$
\mathcal{M}=\left\{u \in \mathcal{D}_{a}^{1, p}(\Omega) \backslash\{0\}:\left\langle I^{\prime}(u), u\right\rangle=0\right\},
$$

Thus, $u \in \mathcal{M}$ if and only if

$$
M\left(\|u\|^{p}\right) \int_{\Omega}|x|^{-a p}|\nabla u|^{p} \mathrm{~d} x-\int_{\Omega}|x|^{-b p^{*}}|u|^{p^{*}} \mathrm{~d} x-\lambda \int_{\Omega} k|u|^{q} \mathrm{~d} x=0
$$

Define

$$
\phi(u)=\left\langle I^{\prime}(u), u\right\rangle
$$


Then, for $u \in \mathcal{M}$

$$
\begin{aligned}
\left\langle\phi^{\prime}(u), u\right\rangle & =p(M)\|u\|^{p}-p^{*} \int_{\Omega}|x|^{-b p^{*}}|u|^{p^{*}} \mathrm{~d} x-\lambda q \int_{\Omega} k|u|^{q} \mathrm{~d} x \\
& =(p-q)(M)\|u\|^{p}-\left(p^{*}-q\right) \int_{\Omega}|x|^{-b p^{*}}|u|^{p^{*}} \mathrm{~d} x \\
& =\left(p-p^{*}\right)(M)\|u\|^{p}+\lambda\left(p^{*}-q\right) \int_{\Omega} k|u|^{q} \mathrm{~d} x .
\end{aligned}
$$

Now, we split $\mathcal{M}$ in three parts:

$$
\begin{aligned}
& \mathcal{M}^{+}=\left\{u \in \mathcal{M}:\left\langle\phi^{\prime}(u), u\right\rangle>0\right\} \\
& \mathcal{M}^{0}=\left\{u \in \mathcal{M}:\left\langle\phi^{\prime}(u), u\right\rangle=0\right\} \\
& \mathcal{M}^{-}=\left\{u \in \mathcal{M}:\left\langle\phi^{\prime}(u), u\right\rangle<0\right\} .
\end{aligned}
$$

Note that $\mathcal{M}$ contains every nontrivial solution of the problem (1.1). Moreover, we have the following results.

Lemma 2. I is coercive and bounded from below on $\mathcal{M}$.

Proof. If $u \in \mathcal{M}$, then by (2.3) and the Hölder inequality, we deduce that

$$
\begin{aligned}
I(u) & =(1 / p) M\left(\|u\|^{p}\right)-\left(1 / p^{*}\right) \int_{\Omega}|x|^{-b p^{*}}|u|^{p^{*}} \mathrm{~d} x-(\lambda / q) \int_{\Omega} k|u|^{q} \mathrm{~d} x \\
& \geq\left(\frac{1}{p}-\frac{1}{p^{*}}\right) M\left(\|u\|^{p}\right)-\lambda\left(\frac{1}{q}-\frac{1}{p^{*}}\right)\|u\|^{q}|k|_{\infty} .
\end{aligned}
$$

Thus, $I$ is coercive and bounded from below on $\mathcal{M}$.

We have the following results.

Lemma 3. Suppose that $u_{0}$ is a local minimizer for $I$ on $\mathcal{M}$. Then, if $u_{0} \notin \mathcal{M}^{0}, \quad u_{0}$ is a critical point of $I$.

Proof. If $u_{0}$ is a local minimizer for $I$ on $\mathcal{M}$, then $u_{0}$ is a solution of the optimization problem

$$
\min _{\{u / \phi(u)=0\}} I(u) .
$$

Hence, there exists a Lagrange multipliers $\theta \in \mathbb{R}$ such that

$$
I^{\prime}\left(u_{0}\right)=\theta \phi^{\prime}\left(u_{0}\right) \text { in } \mathcal{H}^{-1}
$$

Thus,

$$
\left\langle I^{\prime}\left(u_{0}\right), u_{0}\right\rangle=\theta\left\langle\phi^{\prime}\left(u_{0}\right), u_{0}\right\rangle .
$$

But $\left\langle\phi^{\prime}\left(u_{0}\right), u_{0}\right\rangle \neq 0$, since $u_{0} \notin \mathcal{M}^{0}$. Hence $\theta=0$. This completes the proof.

Lemma 4. There exists a positive number $\Lambda_{0}$ such that, for all $\lambda \in\left(0, \Lambda_{0}\right)$ we have $\mathcal{M}^{0}=\varnothing$.

Proof. Let us reason by contradiction.

Suppose $\mathcal{M}^{0} \neq \varnothing$ such that $0<\lambda<\Lambda_{0}$. Moreover, by the Hölder inequality and the Sobolev embedding theorem, we obtain

$$
\|u\| \geq\left(\frac{p-q}{p^{*}-q}\right)^{\frac{1}{p^{*}-p}}\left(S_{a, p}\right)^{\frac{p^{*}}{p\left(p^{*}-p\right)}}
$$


and

$$
\|u\| \leq\left(\frac{p^{*}-q}{p^{*}-p}\right)^{\frac{1}{p-q}} M_{0}^{\frac{-1}{p-q}}|k|_{\infty} \lambda^{\frac{1}{p-q}},
$$

From (2.5) and (2.6), we obtain $\lambda \geq \Lambda_{0}$, which contradicts an hypothesis.

Thus $\mathcal{M}=\mathcal{M}^{+} \cup \mathcal{M}^{-}$. Define

$$
c:=\inf _{u \in \mathcal{M}} I(u), c^{+}:=\inf _{u \in \mathcal{M}^{+}} I(u) \text { and } c^{-}:=\inf _{u \in \mathcal{M}^{-}} I(u) .
$$

For the sequel, we need the following Lemma.

Lemma 5. If $M_{0}>\frac{p}{p^{*}}$ then,

1) For all $\lambda$ such that $0<\lambda<\Lambda_{0}$, one has $c \leq c^{+}<0$.

2) There exists $\Lambda_{1}>0$ such that for all $0<\lambda<\Lambda_{1}$, one has $c^{-}>C_{0}$.

Proof.

1) Let $u \in \mathcal{M}^{+}$. By (2.4), we have

$$
\left(\frac{p-q}{p^{*}-q}\right)\|u\|^{p}>\int_{\Omega}|x|^{-b p^{*}}|u|^{p^{*}} \mathrm{~d} x
$$

and so

$$
\begin{aligned}
I(u) & =\left(\frac{1}{p}-\frac{1}{q}\right) M_{0}\|u\|^{p}+\left(\frac{1}{q}-\frac{1}{p^{*}}\right)|x|^{-b p^{*}}|u|^{p^{*}} \\
& <\left(\frac{p-q}{q}\right)\|u\|^{p}\left(\frac{1}{p^{*}}-\frac{M_{0}}{p}\right) .
\end{aligned}
$$

If $M_{0}>\frac{p}{p^{*}}$, we conclude that $c \leq c^{+}<0$.

2) Let $u \in \mathcal{M}^{-}$. By (2.4) and the Hölder inequality we get

$$
I(u) \geq\left[\left(\frac{1}{p}-\frac{1}{p^{*}}\right) M_{0}\left(\frac{p-q}{p^{*}-q}\right)^{p\left(\frac{p-q}{p^{*}-p}\right)}\left(S_{a, p}\right)^{\frac{p^{*}(p-q)}{\left(p^{*}-p\right)}}-\lambda\left(\frac{1}{q}-\frac{1}{p^{*}}\right)|k|_{\infty}\right]\|u\|^{q} .
$$

Thus, for all $\lambda$ such that

$$
0<\lambda<\Lambda_{1}=\frac{q}{p} M_{0}\left(\frac{p^{*}-p}{p^{*}-q}\right)\left(\frac{p-q}{p^{*}-q}\right)^{p\left(\frac{p-q}{p^{*}-p}\right)}\left(S_{a, p}\right) \frac{p^{*}(p-q)}{p\left(p^{*}-p\right)}|k|_{\infty}^{-1},
$$

we have $I(u) \geq C_{0}$.

For each $u \in \mathcal{D}_{a}^{1, p}(\Omega) \backslash\{0\}$, we write

$$
t_{m}:=t_{\max }(u)=\left[\frac{\left(\frac{p}{q}-1\right) M_{0}\|u\|^{p}}{\left(\frac{p^{*}}{q}-1\right) \int_{\Omega}|x|^{-b p^{*}}|u|^{p^{*}} \mathrm{~d} x}\right]^{1 /\left(p^{*}-p\right)}>0 .
$$

Lemma 6. Let $\lambda$ real parameters such that $0<\lambda<\Lambda_{0}$. For each $u \in \mathcal{D}_{a}^{1, p}(\Omega)$, there exist unique $t^{+}$and $t^{-}$such that $0<t^{+}<t_{m}<t^{-}$, 


$$
\begin{aligned}
\left(t^{+} u\right) \in \mathcal{M}^{+},\left(t^{-} u\right) \in \mathcal{M}^{-} & \\
I\left(t^{+} u\right) & =\inf _{0 \leq t \leq t_{m}} I(t u) \text { and } I\left(t^{-} u\right)=\sup _{t \geq 0} I(t u) .
\end{aligned}
$$

Proof. With minor modifications, we refer to [13].

Proposition 1. (see [13])

1) For all $\lambda$ such that $0<\lambda<\Lambda_{0}$, there exists a $(P S)_{c^{+}}$sequence in $\mathcal{M}^{+}$.

2) For all $\lambda$ such that $0<\lambda<\Lambda_{1}$, there exists a a $(P S)_{c^{-}}$sequence in $\mathcal{M}^{-}$.

\section{Proof of Theorem 1}

Now, taking as a starting point the work of Tarantello [10], we establish the existence of a local minimum for $I$ on $\mathcal{M}^{+}$.

Proposition 2. For all $\lambda$ such that $0<\lambda<\Lambda_{0}$, the functional $I$ has a minimizer $u_{0}^{+} \in \mathcal{M}^{+}$and it satisfies:

1) $I\left(u_{0}^{+}\right)=c=c^{+}$,

2) $\left(u_{0}^{+}\right)$is a nontrivial solution of (1.1).

Proof. If $0<\lambda<\Lambda_{0}$, then by Proposition 1 (1) there exists a $\left(u_{n}\right)_{n}(P S)_{c^{+}}$ sequence in $\mathcal{M}^{+}$, thus it bounded by Lemma 2 . Then, there exists $u_{0}^{+} \in \mathcal{D}_{a}^{1, p}(\Omega)$ and we can extract a subsequence which will denoted by $\left(u_{n}\right)_{n}$ such that

$$
\begin{aligned}
& u_{n} \rightarrow u_{0}^{+} \text {weakly in } \mathcal{D}_{a}^{1, p}(\Omega) \\
& u_{n} \rightarrow u_{0}^{+} \text {weakly in } L^{p^{*}}(\Omega) \\
& u_{n} \rightarrow u_{0}^{+} \text {strongly in } L^{q}(\Omega) \\
& u_{n} \rightarrow u_{0}^{+} \text {a.e in } \Omega
\end{aligned}
$$

Thus, by (3.1), $u_{0}^{+}$is a weak nontrivial solution of (1.1). Now, we show that $u_{n}$ converges to $u_{0}^{+}$strongly in $\mathcal{H}_{0}^{1}(\Omega)$. Suppose otherwise. By the lower semi-continuity of the norm, then either $\left\|u_{0}^{+}\right\|<\liminf _{n \rightarrow \infty}\left\|u_{n}\right\|$ and we obtain

$$
\begin{aligned}
c & \leq I\left(u_{0}^{+}\right)=(1 / p) M\left(\left\|u_{0}^{+}\right\|^{p}\right)-\left(1 / p^{*}\right) \int_{\Omega}|x|^{-b p^{*}}\left|u_{0}^{+}\right|^{p^{*}} \mathrm{~d} x-(\lambda / q) \int_{\Omega} k\left|u_{0}^{+}\right|^{q} \mathrm{~d} x \\
& <\liminf _{n \rightarrow \infty} I\left(u_{n}\right)=c .
\end{aligned}
$$

We get a contradiction. Therefore, $u_{n}$ converge to $u_{0}^{+}$strongly in $\mathcal{H}_{0}^{1}(\Omega)$. Moreover, we have $u_{0}^{+} \in \mathcal{M}^{+}$. If not, then by Lemma 6 , there are two numbers $t_{0}^{+}$and $t_{0}^{-}$, uniquely defined so that $\left(t_{0}^{+} u_{0}^{+}\right) \in \mathcal{M}^{+}$and $\left(t^{-} u_{0}^{+}\right) \in \mathcal{M}^{-}$. In particular, we have $t_{0}^{+}<t_{0}^{-}=1$. Since

$$
\left.\frac{\mathrm{d}}{\mathrm{d} t} I\left(t u_{0}^{+}\right)\right|_{t=t_{0}^{+}}=0 \text { and }\left.\frac{\mathrm{d}^{2}}{\mathrm{~d} t^{2}} I\left(t u_{0}^{+}\right)\right|_{t=t_{0}^{+}}>0 \text {, }
$$

there exists $t_{0}^{+}<t^{-} \leq t_{0}^{-}$such that $I\left(t_{0}^{+} u_{0}^{+}\right)<I\left(t^{-} u_{0}^{+}\right)$. By Lemma 6, we get

$$
I\left(t_{0}^{+} u_{0}^{+}\right)<I\left(t^{-} u_{0}^{+}\right)<I\left(t_{0}^{-} u_{0}^{+}\right)=I\left(u_{0}^{+}\right),
$$

which contradicts the fact that $I\left(u_{0}^{+}\right)=c^{+}$. Since $I\left(u_{0}^{+}\right)=I\left(\left|u_{0}^{+}\right|\right)$and $\left|u_{0}^{+}\right| \in \mathcal{M}^{+}$, then by Lemma 3 , we may assume that $u_{0}^{+}$is a nontrivial nonnegative solution of (1.1). By the Harnack inequality, we conclude that 
$u_{0}^{+}>0$ and $v_{0}^{+}>0$, see for exanmple [12].

\section{Proof of Theorem 2}

Next, we establish the existence of a local minimum for $I$ on $\mathcal{M}^{-}$. For this, we require the following Lemma.

Lemma 7. Assume that $M_{0}>\frac{p}{p^{*}}$ then for all $\lambda$ such that $0<\lambda<\Lambda_{1}$, the functional $I$ has a minimizer $u_{0}^{-}$in $\mathcal{M}^{-}$and it satisfies:

1) $I\left(u_{0}^{-}\right)=c^{-}>0$,

2) $u_{0}^{-}$is a nontrivial solution of $(1.1)$ in $\mathcal{D}_{a}^{1, p}(\Omega)$.

Proof. If $0<\lambda<\Lambda_{1}$, then by Proposition 1 (2) there exists a $\left(u_{n}\right)_{n},(P S)_{c^{-}}$ sequence in $\mathcal{M}^{-}$, thus it bounded by Lemma 2 . Then, there exists $u_{0}^{-} \in \mathcal{D}_{a}^{1, p}(\Omega)$ and we can extract a subsequence which will denoted by $\left(u_{n}\right)_{n}$ such that

$$
\begin{aligned}
& u_{n} \rightarrow u_{0}^{-} \text {weakly in } \mathcal{D}_{a}^{1, p}(\Omega) \\
& u_{n} \rightarrow u_{0}^{-} \text {weakly in } L^{p^{*}}(\Omega) \\
& u_{n} \rightarrow u_{0}^{-} \text {strongly in } L^{q}(\Omega) \\
& u_{n} \rightarrow u_{0}^{-} \text {a.e in } \Omega
\end{aligned}
$$

This implies that

$$
\int_{\Omega}|x|^{-b p^{*}}\left|u_{n}\right|^{p^{*}} \mathrm{~d} x \rightarrow \int_{\Omega}|x|^{-b p^{*}}\left|u_{0}^{-}\right|^{p^{*}} \mathrm{~d} x \text {, as } n \text { goes to } \infty .
$$

Moreover, by $(K)$ and (2.4) we obtain

$$
\begin{aligned}
\int_{\Omega}|x|^{-b p^{*}}\left|u_{n}\right|^{p^{*}} \mathrm{~d} x & >\left[\frac{(p-q)}{\left(p^{*}-q\right)} M_{0}\left(\left\|u_{n}\right\|^{p}\right)\right] \\
& >M_{0}\left(\frac{p-q}{p^{*}-q}\right)^{\left(\frac{p^{*}}{p^{*}-p}\right)}\left(S_{a, p}\right) \frac{p^{*}}{\left(p^{*}-p\right)}=C_{1}
\end{aligned}
$$

if $M_{0}>\frac{p}{p^{*}}$ we get

$$
\int_{\Omega}|x|^{-b p^{*}}\left|u_{n}\right|^{p^{*}} \mathrm{~d} x>C_{1}>0
$$

This implies that

$$
\int_{\Omega}|x|^{-b p^{*}}\left|u_{0}^{-}\right|^{p^{*}} \mathrm{~d} x \geq C_{1} .
$$

Now, we prove that $\left(u_{n}\right)_{n}$ converges to $u_{0}^{-}$strongly in $\mathcal{D}_{a}^{1, p}(\Omega)$. Suppose otherwise. Then, either $\left\|u_{0}^{-}\right\|<\liminf _{n \rightarrow \infty}\left\|u_{n}\right\|$. By Lemma 6 there is a unique $t_{0}^{-}$ such that $\left(t_{0}^{-} u_{0}^{-}\right) \in \mathcal{M}^{-}$. Since

$$
u_{n} \in \mathcal{M}^{-}, I\left(u_{n}\right) \geq I\left(t u_{n}\right), \text { for all } t \geq 0,
$$

we have

$$
I\left(t_{0}^{-} u_{0}^{-}\right)<\lim _{n \rightarrow \infty} I\left(t_{0}^{-} u_{n}\right) \leq \lim _{n \rightarrow \infty} I\left(u_{n}\right)=c^{-},
$$


and this is a contradiction. Hence,

$$
\left(u_{n}\right)_{n} \rightarrow u_{0}^{-} \text {strongly in } \mathcal{D}_{a}^{1, p}(\Omega) .
$$

Thus,

$$
I\left(u_{n}\right) \text { converges to } I\left(u_{0}^{-}\right)=c^{-} \text {as } n \text { tends to }+\infty .
$$

Since $I\left(u_{0}^{-}\right)=I\left(\left|u_{0}^{-}\right|\right)$and $u_{0}^{-} \in \mathcal{M}^{-}$, then by (4.1) and Lemma 3, we may assume that $u_{0}^{-}$is a nontrivial nonnegative solution of (1.1). By the maximum principle, we conclude that $u_{0}^{-}>0$.

Now, we complete the proof of Theorem 2. By Propositions 2 and Lemma 7, we obtain that (1.1) has two positive solutions $u_{0}^{+} \in \mathcal{M}^{+}$and $u_{0}^{-} \in \mathcal{M}^{-}$. Since $\mathcal{M}^{+} \cap \mathcal{M}^{-}=\varnothing$, this implies that $u_{0}^{+}$and $u_{0}^{-}$are distinct.

\section{Proof of Theorem 3}

In this section, we consider the following Nehari submanifold of $\mathcal{M}$

$$
\mathcal{M}_{\varrho}=\left\{u \in \mathcal{D}_{a}^{1, p}(\Omega) \backslash\{0\}:\left\langle I^{\prime}(u), u\right\rangle=0 \text { and }\|u\| \geq \rho>0\right\} .
$$

Thus, $u \in \mathcal{M}_{\rho}$ if and only if

$$
M\left(\|u\|^{p}\right) \int_{\Omega}|x|^{-a p}|\nabla u|^{p} \mathrm{~d} x-\int_{\Omega}|x|^{-b p^{*}}|u|^{p^{*}} \mathrm{~d} x-\lambda \int_{\Omega} k|u|^{q} \mathrm{~d} x=0 \text { and }\|u\| \geq \rho>0 .
$$

Firsly, we need the following Lemmas

Lemma 8. Under the hypothesis of Theorem 3, there exist $\varrho_{0}$ such that $\mathcal{M}_{\varrho}$ is nonempty for any $\lambda<0$ and $\varrho \in\left(0, \varrho_{0}\right)$.

Proof. Fix $u_{0} \in \mathcal{D}_{a}^{1, p}(\Omega) \backslash\{0\}$ and let

$$
\begin{aligned}
g(t) & =\left\langle I^{\prime}\left(t u_{0}\right), t u_{0}\right\rangle \\
& =t^{p} M_{0}\left\|u_{0}\right\|^{p}-t^{p^{*}} \int_{\Omega}|x|^{-b p^{*}}\left|u_{0}\right|^{p^{*}} \mathrm{~d} x-\lambda t^{q} \int_{\Omega} k\left|u_{0}\right|^{q} \mathrm{~d} x .
\end{aligned}
$$

Clearly $g(0)=0$ and $g(t) \rightarrow-\infty$ as $n \rightarrow+\infty$. Moreover, we have

$$
\begin{aligned}
g(1) & =M_{0}\left\|u_{0}\right\|^{p}-\int_{\Omega}|x|^{-b p^{*}}\left|u_{0}\right|^{p^{*}} \mathrm{~d} x-\lambda \int_{\Omega} k\left|u_{0}\right|^{q} \mathrm{~d} x \\
& \geq\left\|u_{0}\right\|^{p}\left[M_{0}-\left(S_{a, p}\right)^{\frac{p^{*}}{p}} h_{0}\left\|u_{0}\right\|^{p^{*}-p}\right]-\lambda\left\|u_{0}\right\|^{q}|k|_{\infty} .
\end{aligned}
$$

If $\left\|u_{0}\right\| \geq \varrho>0$ for $0<\rho<\rho_{0}=M_{0}^{\frac{1}{p^{*}-p}}\left(S_{a, p} \frac{p^{*}}{p\left(p^{*}-p\right)}\right.$, then there exist $\Lambda_{2}=\left(M_{0} \varrho^{p-q}-\left(S_{a, p}\right)^{\frac{-p^{*}}{p}} \varrho^{p^{*}-q}\right)|k|_{\infty}^{-1}$ and $t_{0}>0$ such that $g\left(t_{0}\right)=0$. Thus, $\left(t_{0} u_{0}\right) \in \mathcal{M}_{\varrho}$ and $\mathcal{M}_{\varrho}$ is nonempty for any $\lambda<\Lambda_{2}$.

Lemma 9. There exist $\Lambda_{3}>0$ and $M$ positive real such that

$$
\left\langle\phi^{\prime}(u), u\right\rangle<-M<0 \text {, }
$$

for $u \in \mathcal{M}_{\varrho}$ and any $\lambda<\Lambda_{3}$. 
Proof. Let $u \in \mathcal{M}_{\varrho}$ then by (2.3), (2.4) and the Holder inequality, allows us to write

$$
\left\langle\phi^{\prime}(u), u\right\rangle \leq\|u\|^{q}\left[\lambda\left(p^{*}-q\right)|k|_{\infty}-\left(p^{*}-p\right) M_{0} \varrho^{p-q}\right]
$$

Thus, if $\lambda<\Lambda_{3}=\min \left(\Lambda_{2}, \Lambda_{4}\right)$ with $\Lambda_{4}=\frac{\left(p^{*}-p\right) M_{0} \varrho^{p-q}}{\left(p^{*}-q\right)|k|_{\infty}}$, then we obtain that

$$
\left\langle\phi^{\prime}(u), u\right\rangle<0 \text {, for any } u \in \mathcal{M}_{\varrho} \text {. }
$$

Lemma 10. There exist $\mathrm{r}$ and $\eta$ positive constants such that if $M_{0}>\frac{p}{p^{*}}$,

1) We have

$$
I(u) \geq \eta>0 \text { for }\|u\|_{a}=r .
$$

2) There exists $\sigma \in \mathcal{M}_{\varrho}$ when $\|\sigma\|>r$, with $r=\|u\|$, such that $I(\sigma) \leq 0$.

Proof. We can suppose that the minima of $I$ are realized by $\left(u_{0}^{+}\right)$and $u_{0}^{-}$. The geometric conditions of the mountain pass theorem are satisfied. Indeed, we have

a) By (2.4), (5.1), the Holder inequality, we get

$$
I(u) \geq\left[\left(\frac{1}{p}-\frac{1}{q}\right) M_{0}+\left(\frac{p-q}{p^{*} q}\right)\right]\|u\|^{p} .
$$

Thus, for $M_{0}>\frac{p}{p^{*}}$ there exist $\eta, r>0$ such that

$$
I(u) \geq \eta>0 \text { when } r=\|u\| \text { small. }
$$

b) Let $t>0$, then we have for all $\theta \in \mathcal{M}_{\rho}$

$$
I(t \theta)=\frac{t^{p}}{p} M\left(\|\theta\|^{p}\right)-\frac{t^{p^{*}}}{p^{*}} \int_{\Omega}|x|^{-b p^{*}}|\theta|^{p^{*}} \mathrm{~d} x-\lambda \frac{t^{q}}{q} \int_{\Omega} k|\theta|^{q} \mathrm{~d} x .
$$

Letting $\sigma=t \theta$ for $t$ large enough, we obtain $I(\sigma) \leq 0$. For $t$ large enough we can ensure $\|\sigma\|>r$.

Let $\Gamma$ and $c$ defined by

$$
\Gamma:=\left\{\gamma:[0,1] \rightarrow \mathcal{M}_{\varrho}: \gamma(0)=u_{0}^{-} \text {and } \gamma(1)=u_{0}^{+}\right\}
$$

and

$$
c:=\inf _{\gamma \in \Pi} \max _{t \in[0,1]}(I(\gamma(t)))
$$

\section{Proof of Theorem 3.}

If $0<\lambda<\min \left(\Lambda_{1}, \Lambda_{2}\right)$ then, by the Lemmas 2 and Proposition 1 (2), $I$ verifying the Palais-Smale condition in $\mathcal{M}_{\varrho}$. Moreover, from the Lemmas 3, 9 and 10 , there exists $u_{c}$ such that

$$
I\left(u_{c}\right)=c \text { and } u_{c} \in \mathcal{M}_{\underline{\varrho}} .
$$

Thus $u_{c}$ is the third solution of our system such that $u_{c} \neq u_{0}^{+}$and $u_{c} \neq u_{0}^{-}$. Since (1.1) is odd with respect $u$, we obtain that $-u_{c}$ is also a solution of (1.1). 
Conclusion 1. In our work, we have searched the critical points as the minimizers of the energy functional associated to the problem on the constraint defined by the Nehari manifold $\mathcal{M}$, which are solutions of our problem. Under some sufficient conditions on coefficients of equation of (1.1), we split $\mathcal{M}$ in two disjoint subsets $\mathcal{M}^{+}$and $\mathcal{M}^{-}$thus we consider the minimization problems on $\mathcal{M}^{+}$and $\mathcal{M}^{-}$respectively. In the Sections 3 and 4 we have proved the existence of at least two nontrivial solutions on $\mathcal{M}_{\varrho}$ for all $0<\lambda<\min \left(\Lambda_{1}, \Lambda_{2}\right)$.

In the perspectives we will try to find more nontrivil solutions by splliting again the sub varieties of Nehari.

\section{Acknowledgements}

The author gratefully acknowledge Qassim University, represented by the Deanship of Scientific Research, on the material support for this research under the number (1027) during the academic year 1440AH/2019AD.

\section{Conflicts of Interest}

The authors declare no conflicts of interest regarding the publication of this paper.

\section{References}

[1] Kirchhoff, G. (1883) Mechanik, Teubner, Leipzig, Germany.

[2] Bonanno, G. (2004) A Critical Points Theorem and Nonlinear Differential Problems. Journal of Global Optimization, 28, 249-258. https://doi.org/10.1023/B:JOGO.0000026447.51988.f6

[3] Alves, C.O., Correa, F.J.S.A. and Ma, T.F. (2005) Positive Solutions for a Quasilinear Elliptic Equation of Kirchhoff Type. Computers \& Mathematics with Applications, 49, 85-93. https://doi.org/10.1016/j.camwa.2005.01.008

[4] Cheng, C.T. and Wu, X. (2009) Existence Results of Positive Solutions of Kirchhoff Type Probleme. Nonlinear Analysis: Theory, Methods \& Applications, 71, 4883-4892. https://doi.org/10.1016/j.na.2009.03.065

[5] Ma, T.F. and Rivera, J.E.M. (2003) Positive Solutions for a Nonlinear Nonlocal Elliptic Transmission Problem. Applied Mathematics Letters, 16, 243-248. https://doi.org/10.1016/S0893-9659(03)80038-1

[6] Chen, C., Kuo, Y. and Wu, T. (2011) The Nehari Manifold for a Kirchhoff Type Problem Involving Sign-Changing Weight Functions. Journal of Differential Equations, 250, 1876-1908. https://doi.org/10.1016/j.jde.2010.11.017

[7] Mao, A.M. and Zhang, Z.T. (2009) Sign-Changing and Multiple Solutions of Kirchhoff Type Problems without the P.S. Condition. Nonlinear Analysis: Theory, Methods \& Applications, 70, 1275-1287. https://doi.org/10.1016/j.na.2008.02.011

[8] Mao, A.M. and Luan, S.X. (2011) Sign-Changing Solutions of a Class of Nonlocal Quasilinear Elliptic Boundary Value Problems. Journal of Mathematical Analysis and Applications, 383, 239-243. https://doi.org/10.1016/j.jmaa.2011.05.021

[9] Jin, J.H. and Wu, X. (2010) Infinitely Many Radial Solutions for Kirchhoff-Type Problems in $R^{N}$. Journal of Mathematical Analysis and Applications, 369, 564-574. 
https://doi.org/10.1016/j.jmaa.2010.03.059

[10] Tarantello, G. (1992) On Nonhomogeneous Elliptic Equations Involving Critical Sobolev Exponent. Nonlinear Analysis, 9, 281-304.

[11] Ambrosetti, A. and Rabinowitz, P.H. (1973) Dual Variational Methods in Critical Points Theory and Applications. Journal of Functional Analysis, 14, 349-381. https://doi.org/10.1016/0022-1236(73)90051-7

[12] Brown, K.J. and Zhang, Y. (2003) The Nehari Manifold for a Semilinear Elliptic Equation with a Sign-Changing Weight Function. Journal of Differential Equations, 193, 481-499. https://doi.org/10.1016/S0022-0396(03)00121-9

[13] He, X.M. and Zou, W.M. (2009) Infinitely Many Positive Solutions for Kirchhoff-Type Problems. Nonlinear Analysis. Theory, Methods \& Applications, 70, 1407-1414. https://doi.org/10.1016/j.na.2008.02.021 